\title{
Dual-layer Antifouling Membrane of Electrospun PAN: PMMA Nonwoven Nanofibers for Oily Wastewater Treatment
}

\author{
Sura M. Alkarbouly ${ }^{1}$ and Basma I. Waisi ${ }^{2 *}$ \\ \{Sora.moath@gmail.com__Basmawaisi@coeng.uobaghdad.edu.iq\}
}

Department of Chemical Engineering, College of Engineering, University of Baghdad ${ }^{1,2}$

\begin{abstract}
Antifouling membranes have gained considerable interest in water filtration applications. A dual layer of nonwoven nanofibers membranes was fabricated via the electrospinning method using hydrophilic and hydrophobic polymers. The first (base) layer was hydrophilic polyacrylonitrile (PAN) based electro spun nanofibers. In contrast, the second (top) layer was hydrophobic polymethyl methacrylate (PMMA) based electro spun nanofibers. A series of dual-layer nonwoven nanofiber membranes with various PAN: PMMA amount percentages were fabricated and characterized regarding their morphology and chemical composition using Scanning electron microscopy (SEM) and $\mathrm{X}$-ray diffraction (XRD). The chemical functional groups on the membrane surface were detected using the Fourier transform infrared (FTIR). Also, the roughness and wettability of the membrane surface were measured using the atomic force microscopy (AFM) and the contact angle, respectively. The prepared nanofiber membranes efficiency was examined in emulsified oil/water separation using a crossflow filtration system. The results showed that the fouling resistance and permeate flux were significantly improved by spinning the hydrophobic (PMMA) nanofibers on the surface of the hydrophilic (PAN) nanofibers membrane.
\end{abstract}

Keywords: Electro spun nanofibers, emulsified oil, polyacrylonitrile, polymethyl methacrylate, filtration.

\section{Introduction}

Membrane technology, with its high efficiency and integrity, plays a key role in delivering sustainable water and energy resources [1,2]. Microfiltration (MF) is a membrane-based technique for pre-treating feed for reverse osmosis desalination units or removing colloids, bacteria, and oil from recovered wastewater [3,4]. In general, oil contaminates water in different forms, including emulsified oil which is not easy to remove because of its aqueous phase stability [5]. Emulsions pose particular challenges because of the deformability of the droplets, coalescence in bulk and on the membrane surface, membrane wetting by droplets and films, pore blockage, and oil intrusion [6]. In oil filtration membranes, the structural characteristics and surface qualities are crucial, including the permeable structure and wettability (the ability to separate oil from water) [7-9]. Recently, the membranes' hydrophilicity and hydrophobicity development offer an alternative direction for oil/water separation [9-11]. Polymeric fibres are amongst the most acceptable alternatives for functional materials with regulated porosity and selected wettability $[12,13]$. Electro spun polymeric nanofiber membranes have gotten much attention in recent years because of their unique properties such as the high surface-to-volume ratio, high permeability, the ability to manufacture various materials by chemical alteration, and the ability to create coaxial structures [14,15] [16]. The nanofiber membrane is made using the electrospinning technique, which involves charging a jet of fluid that travels through a 
capillary tube with a high-voltage power source [17]. Because of their great mechanical and chemical resilience, polysulfide (PSU), polyether sulfone (PES), polyvinylidene fluoride (PVDF), and polyacrylonitrile (PAN) are commonly utilised to make nonwoven nanofiber membranes $[15,18]$.

On the other hand, fouling is a common problem for oil-removing membranes, causing a significant reduction in initial membrane hydraulic permeability and durability (19)(20). Membrane fouling formed due to the reversible and irreversible deposition of particles, colloids, macromolecules, salts, and other components. Fouling mitigation approaches can be made in various ways, including membrane surface modification and backwashing. Fouling mitigation on membranes has been suggested using surface functionalization and the application of blending additives to promote surface hydrophilicity or pore architecture [6,21][22]. Incorporating high porosity nanofiber layers also can reduce the contact time between the fouling and filtrating layers [23]. A polymeric mixture of polyethylene terephthalate and polyacrylonitrile has been electro spun to make a nanofibrous composite that serves as a support layer for thin active coatings to increase the membrane fouling resistance and water permeability (24)(25). Also, the electro spun polymeric membrane has been utilized as a porous layer with reduced porosity in thin-film composite nanofiltration membranes to enhance permeability [26].

This project aims to make dual-layer antifouling membranes by electrospinning a top layer of polymethyl methacrylate (PMMA) nonwoven nanofiber on a layer of polyacrylonitrile (PAN) nonwoven nanofiber producing a dual-layer PAN: PMMA nonwoven nanofiber membrane. The fabricated nanofibers membranes were characterized using scanning electron microscopy (SEM), X-ray diffraction (XRD), Atomic force microscopy (AFM), Fourier Transform Infrared (FTIR), water contact angle (WCA), and Porosity. The dual-layer nanofiber membranes efficiency was applied in a crossflow filtration system using the emulsified Kerosene in water as a model of organic pollutant.

\section{Materials and Methods}

\subsection{Materials}

Polyacrylonitrile (PAN) (Mwt. of 150,000 g/mole) and polymethyl methacrylate (PMMA) (Mwt. of 350,000 g/mole) were supplied by Aldrich. N, N-Dimethylformamide (DMF) (density of $0.948 \mathrm{~g} / \mathrm{cm} 3$ ) was supplied from Alfa Aesar and used as a solvent to dissolve PAN. Acetone (ACE) (density of $0.7845 \mathrm{~g} / \mathrm{cm} 3$ ) was ordered from Sigma-Aldrich to be used with DMF in dissolving the PMMA. Kerosene (from midland Iraqi refineries company) was used to prepare the synthetic emulsion solution.

\subsection{Methods}

To prepare $11 \mathrm{wt} . \%$ PAN solutions, a specific amount of PAN was dissolved in DMF and stirred continuously for $4 \mathrm{hr}$ at $60{ }^{\circ} \mathrm{C}$. Also, to prepare a homogeneous precursor solution of $6 \mathrm{wt} . \%$ PMMA solution, a certain amount of PMMA was dissolved in a binary solvent of DMF/ACE (weight ratio of 4:6) using a magnetic stirrer for $6 \mathrm{hr}$ in room temperature. Although DMF is a good solvent to dissolve PMMA, ACE should be added with the DMF to achieve a spun-able precursor solution of PMMA [27]. The DMF solvent demonstrated a low degree of macromolecule chains overlap and aggregation in solutions, leading to lower solution viscosity and formation beads. The ACE, on the other hand, presents a larger degree of macromolecule chain overlap and aggregation in solutions, which stops polymer chains from flowing, favouring increased solution viscosity and continually inhibiting beads formation in manufactured PMMA nanofibers [28].

All the nonwoven nanofibers membranes in this work were fabricated using the electrospinning technique, a pulling motion of polymer droplets to solve the surface tension in a high-voltage 
electrostatic field. The main fabricated nanofiber membranes were a pure $11 \mathrm{wt} . \%$ PAN/DMF and a pure $6 \mathrm{wt} . \%$ PMMA (DMF: ACE). The other fabricated nanofiber membranes were dual layers of different amounts percentages of the two polymers PAN: PMMA (75:25, 50:50, and 25:75). Firstly, the base layer of nonwoven PAN-based nanofibers membranes was fabricated by spinning a certain amount of $11 \mathrm{wt} . \%$ PAN/DMF solution using a voltage of $21 \mathrm{kV}$. Then, a top layer of nonwoven nanofibers was fabricated by spinning a certain amount of $6 \mathrm{wt} . \%$ PMMA/(DMF: ACE) precursor solution using a voltage of $18 \mathrm{kV}$.

For all fabricated membranes, the electrospinning parameters were unchanged: flow rate of 1 $\mathrm{ml} / \mathrm{hr}$, the distance between the needle and collector of $15 \mathrm{~cm}$, collector rotating speed of 70 $\mathrm{rpm}$, and the relative humidity $(10 \%-20 \%)$ at room temperature [29-31].

\subsection{Characterization of membranes}

The surfaces' structure and morphologies of the pure PAN and the pure PMMA nonwoven nanofiber membranes and the dual-layers of PAN: PMMA nonwoven nanofibers before and after oil removal were visualized. The samples were coated with a thin layer of gold by sputtering for 80 seconds to acquire a clear surface picture of the membrane surface by field emission scanning electron microscopy (FE-SEM, mara-3, TESCAN). The average fibre size of the PAN and PMMA nonwoven nanofibers was calculated using Image $J$ software from SEM images [30]. The Fourier Transform Infrared (FTIR) and energy-dispersive X-ray spectroscopy (EDX) were also utilised to identify the chemical components of the membranes. The atomic force microscope (AFM) was used to evaluate the surface roughness of the nonwoven fibre membrane samples. The contact angles of the nonwoven nanofiber membrane samples were measured at room temperature using a CAM (CAG-20, Jikan). The values were taken as an average of five points after 10 seconds with $5 \pm 0.5 \mu \mathrm{L}$ water droplet volume. The membrane's porosities were measured using the following method. Each nanofibers membrane sample was weighed and recorded as dry weight. Next, the nanofibers membranes were immersed in distilled water for $1 \mathrm{hr}$. Then, the membrane sample was weighted after 30 minutes from removing them from the distilled water. Then porosity by the nanofibers membranes was calculated using equation 1 (32):

Porosity $\left(\varepsilon_{m}\right)=\frac{\left(W_{1}-W_{2}\right)}{A * t * \rho}$

Where the porosity of the membrane is $(\varepsilon), \mathrm{W}_{1}$ is the weight of the wet membrane, $\mathrm{W}_{2}$ is the weight of the dry membrane. A, $t$, and $\rho$ are the effective membrane area, membrane thickness, and water density at room temperature, respectively.

\subsection{Emulsion Separation Performance}

To prepare a stable emulsion solution of $250 \mathrm{mg} / \mathrm{l}$ concentration (the feed solution), Kerosene was mixed with distilled water using Hielscher ultrasonic processor (Hielscher UP400s, Teltow, Germany) at 10,000 rpm for 10 minutes at room temperature $(25-30){ }^{\circ} \mathrm{C}$. The feed emulsion was pumped to the crossflow filtration module cell using a pump. The effective filtration membrane area was $20 \mathrm{~cm}^{2}$. The permeate flow containing the passing components flew through the membrane. The Permeate flux and its oil content concentration were measured over experiment time $2 \mathrm{hr}$ in each trial using a UV-vis spectroscopy based on the absorbance at a wavelength of $196 \mathrm{~nm}$. The permeation flux (J) and rejection rate (R) were calculated according to the following formulas 1 and 2 [33]:

$$
\begin{aligned}
& J=\frac{V}{A * t} \\
& R \%=\left(1-\left(\frac{C_{p}}{C_{\mathrm{f}}}\right)\right) * 100
\end{aligned}
$$


where $\mathrm{J}, \mathrm{A}$, and $\mathrm{t}$ are the volume of permeated water, membrane effective area, measurement time, respectively. And $\mathrm{R}$ is the oil rejection and $\mathrm{C}_{\mathrm{f}}$ and $\mathrm{C}_{\mathrm{p}}$ are the oil concentrations in feed and permeate solutions, respectively.

\section{Results and Discussion}

\subsection{Membranes characterizations}

Adding to the surface membrane SEM images of the fabricated pure $11 \mathrm{wt} \%$ PAN/DMF and pure 6 wt.\% PMMA/(DMF: ACE) based nonwoven nanofibers membranes, the images of surface morphologies of the various amounts of the prepared dual-layers (PAN: PMMA) nonwoven nanofibers membranes are shown in Figure1. The images indicated that both 11 wt.\% PAN/DMF and 6 wt.\% PMMA/(DMF: ACE) membranes were successfully fabricated and composed of long, free of beads, homogeneous and continuous nanofibers with average fibre diameters of 200 and $800 \mathrm{~nm}$, respectively.

The various dual-layers (PAN: PMMA) nonwoven nanofibers membranes ((75:25), (50:50), and (25:75)) showed a mixture of fibre sizes confirming the existence of a layer of PAN and a layer of PMMA in the membrane sample. PAN-based fibbers' small size appeared in the bottom layer of the membrane, where the larger size of PMMA based fibres formed a continuous top layer above the PAN layer. Increasing the ratio of the PMMA in the dual-layer membrane increases the number of the large fibres in the dual-layer membrane.

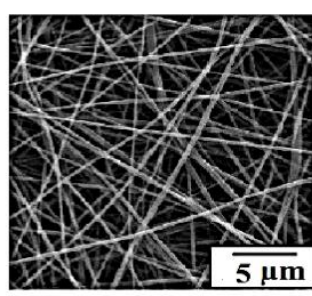

PAN

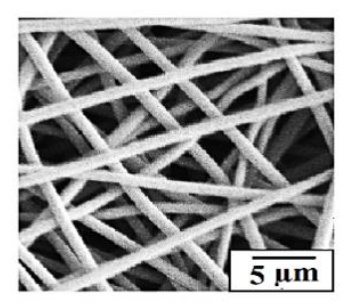

PMMA

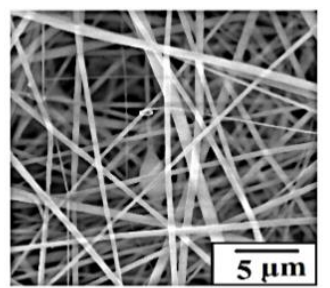

PAN:PMMA (75:25)

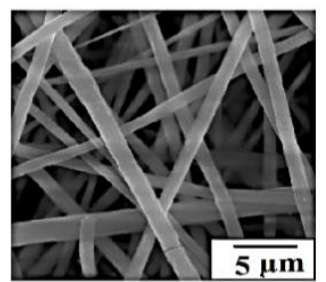

PAN:PMMA (50:50)

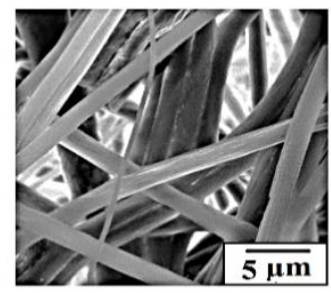

PAN:PMMA (25:75)

Fig 1. The surface SEM images of the various fabricated nanofibers membranes.

Table 1. The EDX analysis results of the various fabricated nanofibers membranes.

\begin{tabular}{ll} 
Element PAN & $75: 25$ \\
\hline & \\
PAN:PMMA & $50: 50$ \\
\hline
\end{tabular}

PAN:PMMA 25:75 PAN:PMMA PMMA

\begin{tabular}{lccccc}
$\mathrm{C}$ & 77.03 & 78.12 & 76.10 & 74.15 & 94.50 \\
\hline $\mathrm{N}$ & 19.63 & 0.00 & 0.00 & 0.00 & 0.00 \\
\hline
\end{tabular}




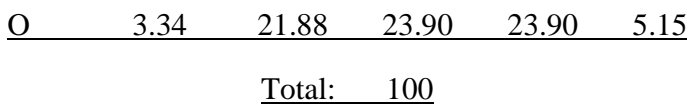

Adding to the surface morphology, the roughness also influences the membrane fouling, which can be characterized using atomic force microscopy (AFM) analyser and showed in Table 2. The average roughness of the fabricated nonwoven nanofiber membrane based on pure PAN recorded a larger value than that of the PMMA base membrane. As a result, in the fabricated dual-layer nanofiber membrane, increasing the amount of PMMA based nanofiber (the top layer) led to a decrease in the surface membrane roughness. In other words, the existing several PAN-based nanofibers in the membrane structure resulted in increasing the roughness of the membrane.

The measured water contact angles also were summarized in Table 2. The PAN-based nanofiber membrane recorded the lowest value because of its high hydrophilicity. In contrast, the PMMA based nanofiber membrane showed the highest contact angle due to its high hydrophobicity (34). As a result, spinning a top layer of the high hydrophobic PMMA based nanofibers $\left(110^{\circ}\right)$ on the layer of high hydrophilic PAN-based nanofibers $\left(33^{\circ}\right)$ caused a hydrophobic dual-layer nanofiber membrane (around $88^{\circ}$ ). Existing hydrophilic and hydrophobic nanofibers in the same membrane resulted in trapping the water in the micro and rough nanoscale structure due to the hydrophilicity and catching the oil droplets inside the hydrophobic fibres instead of clogging the membrane pores.

The other important characterization is the membrane porosity measured depending on water uptake for all fabricated membranes and showed in Table 2 too. The PAN and PMMA based nanofibers showed the highest and lowest porosities, respectively, which can be attributed to the big difference in their hydrophilicity. Existing the hydrophilic PAN-based nanofibers in the dual-layers nanofibers membranes resulted in high porosities. The porosity increases as the PAN content increases, which could be attributed to the nonwoven nanofibers membranes' decreasing average fibre diameter [35].

Table 2. The measured values of average roughness (Ra), porosity, and contact angle of the fabricated nanofiber membranes

\begin{tabular}{llll}
\hline $\begin{array}{l}\text { Samples } \\
\text { name }\end{array}$ & $\begin{array}{l}\text { Roughness } \\
\text { (Ra) } \mathbf{n m}\end{array}$ & $\begin{array}{l}\text { Porosity } \\
(-)\end{array}$ & $\begin{array}{l}\text { Contact } \\
\text { angle } \\
\left(\mathbf{o}^{\mathbf{9}}\right)\end{array}$ \\
\hline $\begin{array}{l}\text { PAN } \\
\text { (75:25) }\end{array}$ & 108.4 & 94 & 33 \\
$\begin{array}{l}\text { PAN:PMMA } \\
\begin{array}{l}\text { (50:50) } \\
\text { PAN:PMMA }\end{array}\end{array}$ & 59.28 & 94 & 83 \\
$\begin{array}{l}\text { (25:75) } \\
\text { PAN:PMMA }\end{array}$ & 60.15 & 95 & 88 \\
PMMA & 60.88 & 96 & 95 \\
\hline
\end{tabular}

FTIR spectrum study of pure PAN and pure PMMA based nanofiber membranes defining functional groups is shown in Fig 2. The apparent sharp, intense peak at $1732.08 \mathrm{~cm}-1$ in the analysis of PMMA based nanofibers is due to ester carbonyl group stretching vibration. The C$\mathrm{O}$ (ester bond) stretching vibration is responsible for the broad peak extending from 1250$1149.57 \mathrm{~cm}-1$. The broadband from 990 to $420 \mathrm{~cm}-1$ is also due to $\mathrm{C}-\mathrm{H}$ bending. Stretching vibration is responsible for the broad peak ranging from 3523-2950 cm-1[36].

Due to the $\mathrm{CN}$ stretching band stretching of the acrylonitrile unit in the polymer chain, the most recognisable peak in the displayed FTIR analysis of PAN-based nanofibers in Fig 2 was seen at $2245.14 \mathrm{~cm} 1$ [37]. $\mathrm{C}=\mathrm{O}$ stretching and $\mathrm{CH}$ stretching aliphatic $\mathrm{CH}$ groups are allocated to the other peaks at 1666.5 and $1373.32 \mathrm{~cm} 1$, respectively [38]. The stretching frequencies of the (C-O) groups, which are coupled to the carbon atoms of the 1, 2, 3-propanetriol and the succinimide ester $(\mathrm{C}-\mathrm{O})$, are associated with the peaks at 1097.50 and $1066.67 \mathrm{~cm} 1$. C-N 
stretch peaks, $\mathrm{CH} 2$ bending, $\mathrm{C}=\mathrm{O}$ stretching, $\mathrm{CH}$ band, and the stretching vibrations of the $-\mathrm{OH}$ bond emerged from absorbed water at $1236.37,1452.40,1737.86,2926.31$, and $3441.01 \mathrm{~cm}-$ 1 , respectively $[37,39,40]$.

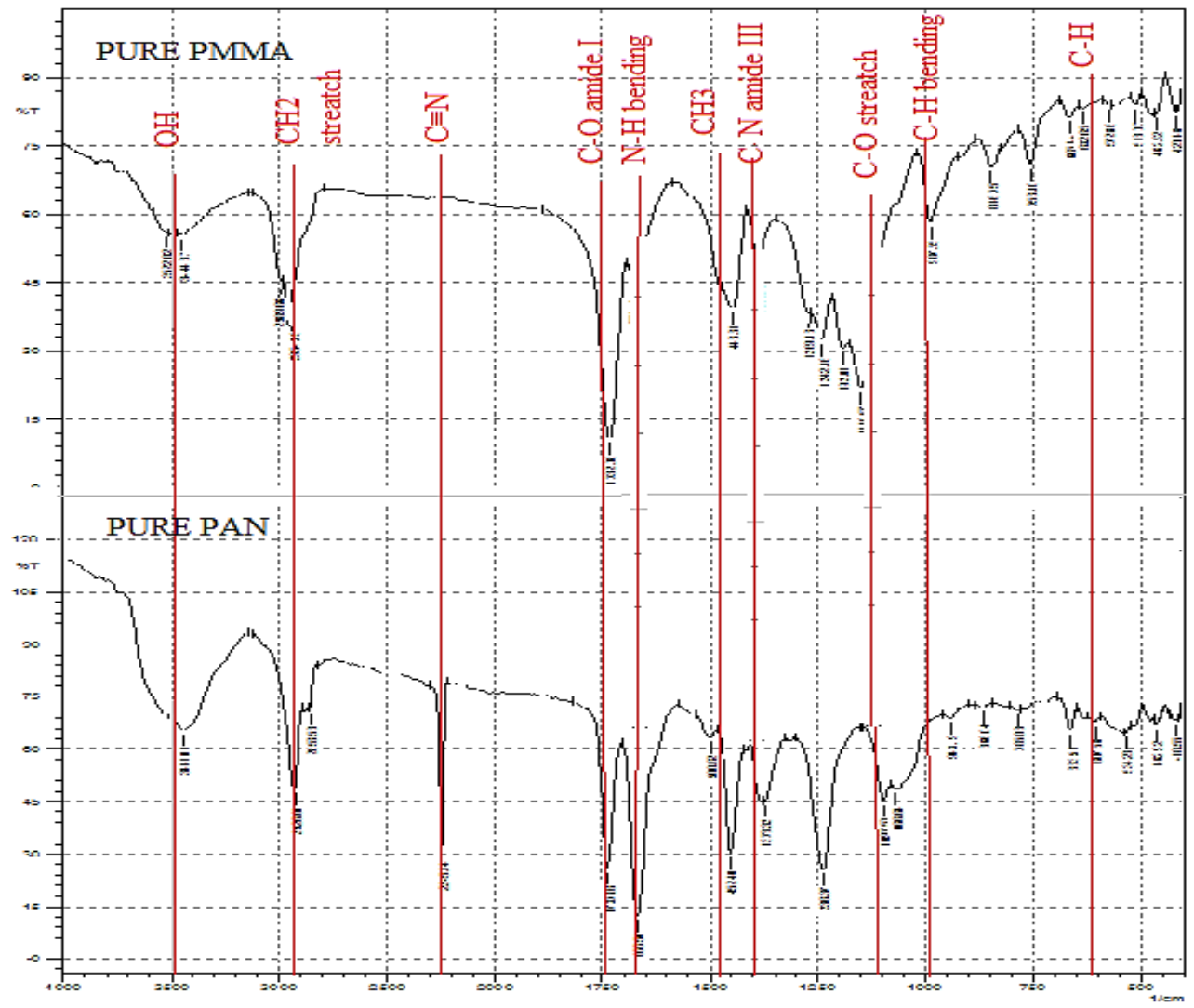

Fig. 2 The Fourier Transform Infrared (FTIR) for pure PAN and PMMA nanofiber membranes.

\section{2 Filtration performance}

The fabricated membrane's efficiency in removing the emulsified oil from water according to the permeate flux and oil rejection percentage is shown in Fig 3 a and b, respectively. The pure PAN-based nanofiber membrane showed a low permeate flux (900 LMH) and high oil rejection percentage $(96 \%)$ due to the accumulated oil droplets on the membrane surface, and clogged membrane pores permeate flux passage, as shown in Fig 4. However, the pure PMMA based nanofiber membrane revealed a much higher permeate flux which can be attributed to the large pore's sizes due to the large size of the PMMA based fibres. Also, the high hydrophobicity surface of PMMA based nanofiber implies a high oil affinity and avoids the formation of an oil fouling layer on the top surface of the membrane.

According to Figs 3 and 4, the dual-layer PAN: PMMA nanofiber membranes also showed a high permeate flux and low fouling participation due to the high hydrophobicity of the membrane surface that wicked the oil droplets into the fibre matrix and decreased the fouling layer on the surface [20]. Incorporating the electro spun PMMA based nanofiber layer did not offer significant additional hydraulic resistance, and hence the electro spun material did not restrict the pores of the PAN-based nanofiber layer. The high permeability obtained using hydrophilic and hydrophobic nanofiber membranes resulted in hydrophilic pores interacting with the water molecules and facilitating water flux. At the same time, the hydrophobic pores 
interact with the oil droplets and decrease the organic layer accumulation on the membrane surface [41][42].

The oil rejection results of the various fabricated nanofibers membranes ranged from $80 \pm 0.7$ $\%$ for pure PMMA to $95.5 \pm 0.6 \%$ for pure PAN membranes. The dual-layers nanofibers membranes showed a reasonable oil rejection (> 87\%) with a slight difference between the various dual-layers membrane. Consequently, incorporating the electro spun layer of PMMA affects the membrane efficiency as expected, considering the PMMA membrane hydrophobicity and pore sizes. The extracted oil droplets were attracted to the hydrophobic surface of the top layer in the dual-layer membrane instead of accumulating on the surface, resulting in an almost free fouling layer, as shown in Fig 4.

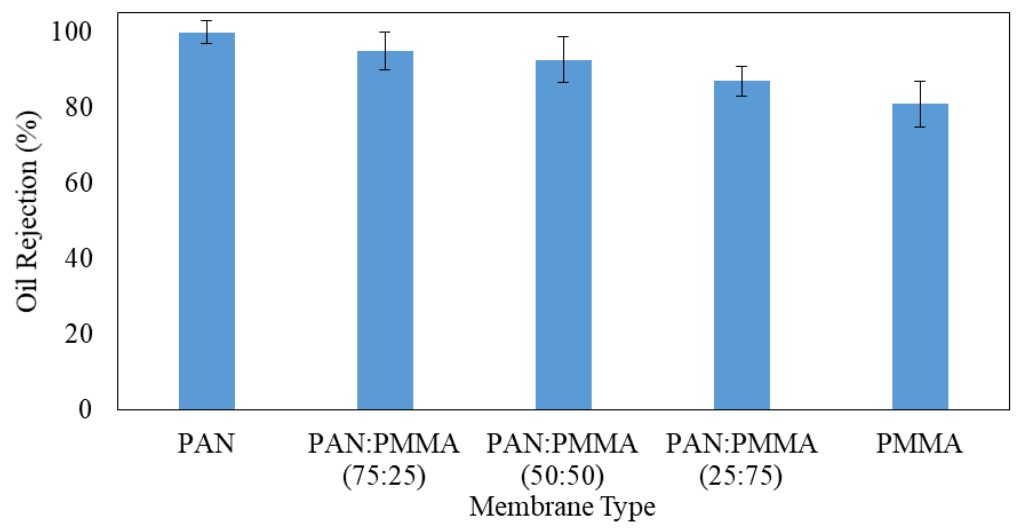

(a)

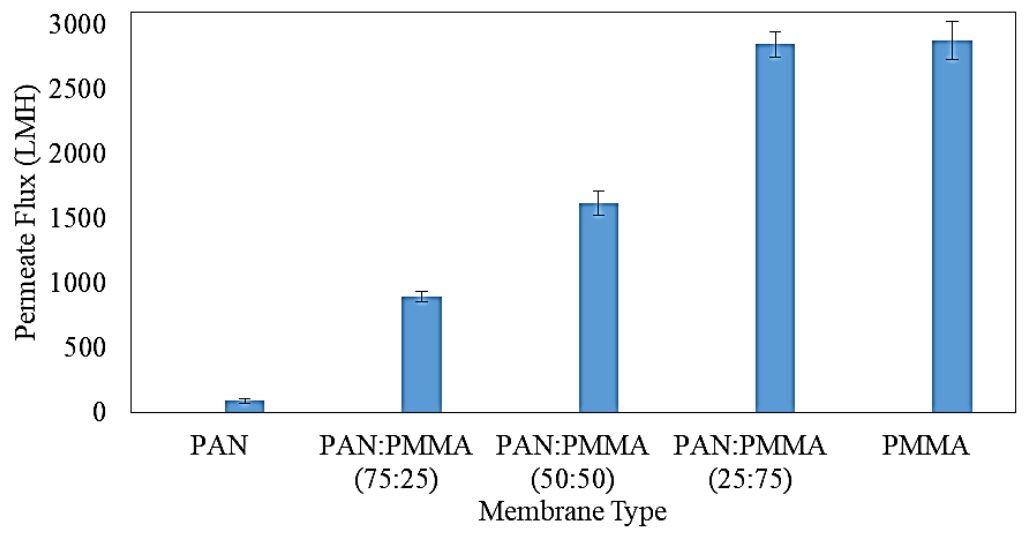

(b)

Fig. 3 Oil/water filtration efficiency using the various fabricated nanofibers membranes (a) permeate flux and (b) oil rejection percentage.

Adding to the hydrophobicity of the nanofiber's surface effect on the fouling formation, the roughness plays an important role. The collection of oil at the valley of the rough surface increases the fouling tendency as the roughness increases [43]. The influence of surface roughness on membrane fouling appears to be reliant on the size of oil droplets in relation to the roughness's characteristic length. The fouling tendency decreases if the oil droplets are much larger than this characteristic length. On the other hand, if the oil droplets are small in comparison to the roughness, they may become caught in the rough surface's valley, resulting in oil collection and membrane fouling [44]. 


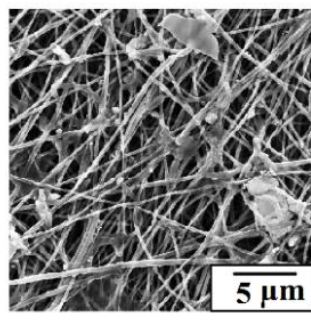

PAN

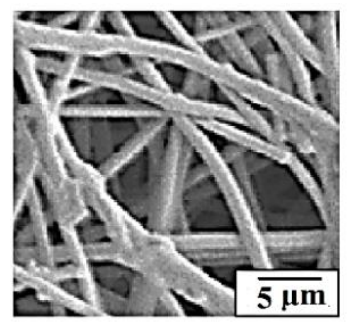

PMMA

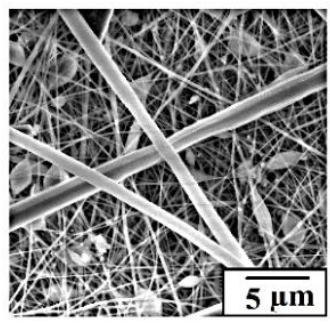

PAN:PMMA (75:25)

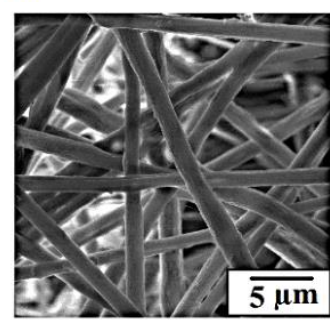

PAN:PMMA (50:50)

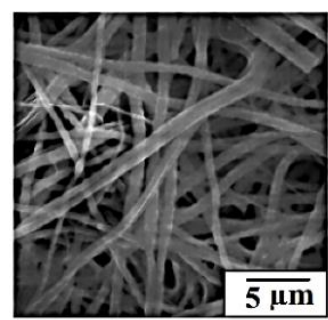

PAN:PMMA (25:75)

Fig. 4 various SEM images fabricated nano-membranes following oil filtration process.

\section{Conclusion}

In this work, dual-layer nanofiber membranes with high organic fouling resistance were successfully fabricated via the electrospinning technique. The fabricated membrane consisted of a top layer of the hydrophobic PMMA nanofibers that spun on the hydrophilic PAN nanofibers membrane base layer. The characterization analysis of the fabricated membranes showed the high effect of the spinning nanofiber layer of PMMA on the base layer of PAN, including the fibre size, porosity, wettability, and roughness. The various prepared membranes were tested in emulsified oil removal using a crossflow filtration system. The dual-layer nanofibers membranes showed a significant improvement in the permeate flux and fouling resistance. Spinning hydrophobic nanofibers of PMMA upon the hydrophilic PAN membrane surface resulted in increasing the permeate flux, which can be argued to the large fibre size (800 $\mathrm{nm}$ ) of the top layer helped the water droplets to flow through easier and cross to the small fibres $(200 \mathrm{~nm})$ of the hydrophilic layer. At the same time, the high hydrophobicity of PMMA nanofibers surface interacts with the oil droplets inside the fibres leading to an almost free fouling membrane.

\section{Reference}

1. Elimelech M, Phillip WA. The Future of Seawater Desalination: Energy, Technology, and the Environment. Science (80- ). 2011;333:712-7.

2. Rajasulochana P, Preethy V. Comparison on efficiency of various techniques in treatment of waste and sewage water-A comprehensive review. Resour Technol. 2016;2(4):175-84.

3. Chakrabarty B, Ghoshal AK, Purkait MK. Ultrafiltration of stable oil-in-water emulsion by polysulfone membrane. J ofMembrane Sci. 2008;325:427-37.

4. Waisi BIH, Karim UF a., Augustijn DCM, Al-Furaiji MHO, Hulscher SJMH. A study on the quantities and potential use of produced water in southern Iraq. Water Sci Technol Water Supply. 2015;15(2):370.

5. Ibrahim S, Wang S, Ang HM. Removal of emulsified oil from oily wastewater using agricultural waste barley straw. Biochem Eng J. 2010;49(1):78-83. 
6. Tummons E, Han Q, Tanudjaja HJ, Hejase CA, Chew JW, Tarabara V V. Membrane fouling by emulsified oil: A review. Sep Purif Technol. 2020;248:116919.

7. Ma W, Zhang Q, Hua D, Xiong R, Zhao J, Rao W, et al. Electrospun fibers for oil-water separation. Vol. 6, RSC Advances. Royal Society of Chemistry; 2016. p. 12868-84.

8. Ying Q, Lili Z, Haixiang S, Li Peng. PAN / PS elctrospun fibers for oil spill cleanup PAN / PS elctrospun fibers for oil spill cleanup. IOP Conf Ser Mater Sci Eng. 2014;62:7.

9. Jeyakumar N, Jeyakumar N, Program M, Seering W. Signature redacted _ Signature redacted Signat-ure redacted. 2017;(2000).

10. Zeiger C, Kumberg J, Vüllers F, Worgull M, Hölscher H, Kavalenka MN. Selective filtration of oil/water mixtures with bioinspired porous membranes. RSC Adv. 2017;7(52):32806-11.

11. Salahi A, Noshadi I, Badrnezhad R, Kanjilal B, Mohammadi T. Nano-porous membrane process for oily wastewater treatment: Optimization using response surface methodology. J Environ Chem Eng. 2013;1(3):218-25.

12. Rajkiewicz M. CHEMICAL AND APPLIED ENGINEERING MATERIALS Interdisciplinary Research and Methodologies www.EngineeringBooksLibrary.c. Canada: Apple Academic Press, Inc; 2015.362 p.

13. Zoka L. Effect of Surface Modification With Electrospun Nanofibers on The Performance of The Ultrafiltration Membrane. 2018.

14. Li D, Xia Y. Electrospinning of nanofibers: Reinventing the wheel? Adv Mater. 2004;16(14):1151-70.

15. Suja PS, Reshmi CR, Sagitha P, Sujith A. Electrospun Nanofibrous Membranes for Water Purification. Polym Rev. 2017;57(3):467-504.

16. Toriello M, Afsari M, Shon HK, Tijing LD. Progress on the fabrication and application of electrospun nanofiber composites. Membranes (Basel). 2020;10(9):1-35.

17. Alghoraibi I, Alomari S. Different methods for nanofibers design and fabrication. Handb Prop Text Tech Fibres. 2019;(February 2018):47.

18. Al-furaiji M, Arena JT, Ren J, Benes N, Nijmeijer A. Triple-Layer Nanofiber Membranes for Treating High Salinity Brines Using Direct Contact Membrane Distillation. Membranes (Basel). 2019;9(60):2-10.

19. Shu W, Liangyin CHU, Wenmei C. Fouling-resistant Composite Membranes for Separation of Oil-in-water Microemulsions. Chinese J ChemEng. 2006;14(1):37-45.

20. Waisi B, Arena JT, Benes NE, Nijmeijer A, McCutcheon JR. Activated Carbon Nanofiber Nonwoven for Removal of Emulsified Oil from Water. Microporous Mesoporous Mater. 2020;296:109966.

21. Abdelrasoul A, Doan H. Ultrasound for membrane fouling control in wastewater treatment and protein purification downstream processing applications. In: Advances in Membrane Technologies. IntechOpen; 2020.

22. Gao H, Sun X, Gao C. Antifouling polysulfone ultrafiltration membranes with sulfobetaine polyimides as novel additive for the enhancement of both water flux and protein rejection. $\mathbf{J}$ Memb Sci. 2017;542:81-90.

23. Karbownik I, Rac-Rumijowska O, Fiedot-Toboła M, Rybicki T, Teterycz H. The preparation and characterization of polyacrylonitrile-polyaniline (PAN/PANI) fibers. Materials (Basel). 2019;12(4):20.

24. Yoon K, Kim K, Wang X, Fang D, Hsiao BS, Chu B. High flux ultrafiltration membranes based on electrospun nanofibrous PAN scaffolds and chitosan coating. Polymer (Guildf). 2006;47(7):2434-41.

25. Hoover LA, Schiffman JD, Elimelech M. Nanofibers in thin-film composite membrane support 
layers: Enabling expanded application of forward and pressure retarded osmosis. Desalination. 2013;308:73-81.

26. Lu T-D, Chen B-Z, Wang J, Jia T-Z, Cao X-L, Wang Y, et al. Electrospun nanofiber substrates that enhance polar solvent separation from organic compounds in thin-film composites. J Mater Chem A. 2018;6(31):15047-56.

27. Ahmad B, Ameer Hamza, Ahmed S, Najam Z, Ishtiaq A. Synthesis and Characterization of PMMA Nanofibers for Filtration of Drinking Water. J Mech Contin Math Sci. 2019;14(4):10216.

28. Gohari RJ, Lau WJ, Matsuura T, Ismail AF. Effect of surface pattern formation on membrane fouling and its control in phase inversion process. J Memb Sci. 2013;446:326-31.

29. Wang R, Liu Y, Li B, Hsiao BS, Chu B. Electrospun nanofibrous membranes for high flux microfiltration. J Memb Sci. 2012;392-393:167-74.

30. Waisi BI, Manickam SS, Benes NE, Nijmeijer A, McCutcheon JR. Activated Carbon Nanofiber Nonwovens: Improving Strength and Surface Area by Tuning the Fabrication Procedure. Ind Eng Chem Res. 2019;58:4084-9.

31. Guo JW, Wang CF, Chen SH, Lai JY, Lu CH, Chen JK. Highly efficient self-cleaning of heavy polyelectrolyte coated electrospun polyacrylonitrile nanofibrous membrane for separation of oil/water emulsions with intermittent pressure. Sep Purif Technol. 2020;234(July 2019):116106.

32. Abdel-aty AAR, Aziz YSA, Ahmed RMG, Elsherbiny IMA, Panglisch S, Ulbricht M, et al. High Performance Isotropic Polyethersulfone Membranes for Heavy Oil-in-Water. J Pre-proofs. $2020 ; 117467$.

33. Wang J-J, Yang $\mathrm{H}-\mathrm{C}$, Wu M-B, Xi Zhang and Zhi-Kang Xu. Nanofiltration Membranes with Cellulose Nanocrystals as an Interlayer for Unprecedented Performance. Mater Chem A. 2017;7.

34. Liu Z, Zhao JH, Liu P, He JH. Tunable surface morphology of electrospun PMMA fiber using binary solvent. Appl Surf Sci. 2016;364:516-21.

35. Yanılmaz M, Zhang X. Polymethylmethacrylate/Polyacrylonitrile Membranes via Centrifugal Spinning as Separator in Li-Ion Batteries. Polymers (Basel). 2015 Apr;7:629-43.

36. Vijayakumari G, Selvakumar N, Jeyasubramanian K, Mala R. Investigation on the electrical properties of polymer metal nanocomposites for physiological sensing applications. Phys Procedia. 2013;49(December):67-78.

37. Shuker H. Antibacterial Activity of Chitosan/PAN Blend Prepared at Different Ratios. AIP Conf Proc. 2019 Dec; 2190.

38. Nithya, S., Selvasekarapandian, S., Premalatha, M. Synthesis and characterization of protonconducting polymer electrolyte based on polyacrylonitrile (PAN). Ionics (Kiel). 2017;23(10):2767-74.

39. Abdouss M, Shoushtari A, Haji A, Moshref B. Fabrication of chelating diethylenetriaminated PAN micro-and nano-fibers for heavy metal removal. Chem Ind Chem Eng Q. 2012 Jan;18:2734.

40. Ulianas A, Heng LY, Ahmad M. A biosensor for urea from succinimide-modified acrylic microspheres based on reflectance transduction. Sensors. 2011;11(9):8323-38.

41. Habimana O, Semião AJC, Casey E. The role of cell-surface interactions in bacterial initial adhesion and consequent biofilm formation on nanofiltration/reverse osmosis membranes. $\mathbf{J}$ Memb Sci. 2014;454:82-96.

42. Yu W, Brown M, Graham NJD. Prevention of PVDF ultrafiltration membrane fouling by coating MnO 2 nanoparticles with ozonation. Sci Rep. 2016;6(1):1-12.

43. Jayalakshmi A, Kim IC, Kwon YN. Cellulose acetate graft-(glycidylmethacrylate-g-PEG) for modification of AMC ultrafiltration membranes to mitigate organic fouling. RSC Adv. 2015;5(60):48290-300. 
44. Huang S, Ras RHA, Tian X. Antifouling membranes for oily wastewater treatment: Interplay between wetting and membrane fouling. Curr Opin Colloid Interface Sci. 2018;36:90-109. 